\title{
The study of the process of chip segmentation in case of edge cutting machining of titanium alloy
}

\author{
Dmitry Sergeevich Ponyatov ${ }^{1}$, Vyacheslav Petrovich Alekseev ${ }^{1, *}$, and Alexandr Isaakovich Khaimovich ${ }^{1}$ \\ ${ }^{1}$ Samara University, 443086 Moskovskoye shosse 34, Russia
}

\begin{abstract}
This article presents the results of the studies of influence of the destruction criterion on the process of segmentation of chips in the process of treatment of VT6 titanium alloy. A series of experiments has been conducted in order to measure the cutting force, the geometry of actual chips, and the experiment's results have been analyzed and processed. Using DEFORM 2D software suite they have developed an imitation model of the milling process which is necessary for the analysis of the impact of cutting conditions on the process of chip formation.
\end{abstract}

\section{Introduction}

In the past several years due to the intensive growth of the technologies of metal processing using cutting it has become topical to study the processes of plastic deformation in the cutting edge area. Destruction of materials in the process of their cutting, i.e. separation of the surface layer from the main body of the workpiece processed is preceded by an intensive plastic deformation of the layer separated and the surface layer. The process of chip formation is accompanied by high effective velocities of deformation which are localized in the area of nominal shearing plane. The higher the level of localization of the focus of plastic deformation the less stressed is the area of chips outside of this focus. [1].

In the process of cutting the most intensive plastic deformation is observed in the small area near the cutting surface sized no more than $0.5 \mathrm{~mm}$, while the velocities of deformation are around $10^{6}$ exponential order and the temperature differential reaches $1,000^{\circ} \mathrm{C}$. An adequate measurement of the actual situation with strain-stress state (SSS) and the temperature in the area with such small dimensions causes significant difficulties therefore the verification means for numerical imitational modeling are almost the only single source of information about the processes in the area of chip formation. Advanced numerical methods of calculation of the strain-stress state enable us to reduce the number of field tests in order to define the temperature and energy parameters of cutting depending on the modes and geometry of the relevant cutting tool [2].

In the course of the past several years the analysis using finite-element method (FEM) has become the main tool for modeling the cutting process [3-9]. T. Thepsonthi, T. Ozel used the FEM analysis in order to study the impact of a cutting tool with CBN coating for processing of Ti$6 \mathrm{Al}-4 \mathrm{~V}$ titanium alloy. The modeling results have been additionally used for the evaluation of the tool's service life. M. Calamaz, D. Coupard, F. Girot have analyzed the impact of the behavior pattern of the material for $\mathrm{KE}$ modeling during chip formation when processing titanium alloys. The modified flow model by JohnsonCooke accounts for the impact of deformation, velocity of deformation, and temperature on the flow stress, as well as introduces the effect of softening deformation. Using the finite-element analysis method Lorentzon, J, Järvstråt, N. Josefson, B.L. have studied the impact of various criteria of destruction on the process of chip formation, placing emphasis on the formation of segmented chips in the process of treatment of Inconel 718 alloy. It has been identified that transition from continuous chip formation to segmented formation had been stipulated by the heat factor and the destruction criterion.

In many studies [10-14] the adequacy of imitational modeling of chip formation in CAE system is determined using temperature- and energy-power parameters. In the process of a field test it is not always possible to adequately identify temperature- and energy-power parameters in the cutting area; therefore one of the indicators of evaluation of the adequacy of imitational modeling is morphology of the chips. The adequacy of imitational modeling using the chip morphology depends on the choice of the corresponding criterion of destruction which determines the moment of the material's exhaustion of plasticity in the course of deformation. As soon as the criterion reaches its peak value the material gets deformed.

The objective of this work is to increase the accuracy of numerical modeling of the cutting process using evaluation of morphology of chip formation in the process of treatment of VT-6 titanium alloy.

\section{Experimental studies}

In order to analyze the geometry of chips and energypower parameters they have used the previous

\footnotetext{
Corresponding author alexeev v.p@,mail.ru
} 
experimental studies [15]. For the experiment they used the workpieces made of VT-6 titanium alloy with the dimensions $\mathrm{L} \times \mathrm{H} \times \mathrm{D} 40 \times 24 \times 6(\mathrm{~mm})$. The surface of each workpiece (sample) has been preliminarily treated to ensure that the initial roughness of all the samples is the same - Ra 1.25.

The experimental studies have been performed using a CNC machine ALZMETALLBAZ 15 CNC equipped with a Kistler dynamometric table which registered changes which comprised the cutting force Fx, Fy, and Fz in real time. The cutting tool was $\varnothing 10 \mathrm{~mm}$ end milling cutter with 4 (Four) teeth.

In order to study the chip shape and its structure polished specimen of the chips have been prepared. In order to prepare micro polished specimen of the chips they used epoxide compound as filler. For etching of the samples they used the following solutions: $\mathrm{HCl}-22$; HNO3 - 15; HF - 41; H2O - 22. In order to study the microstructure they used METAM 1B-32 microscope. The cross sections of the chip segment with the main geometric features are shown in Fig. 1.

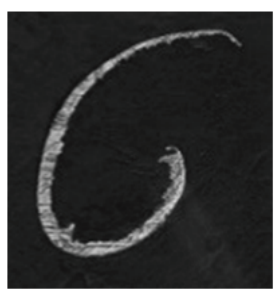

a

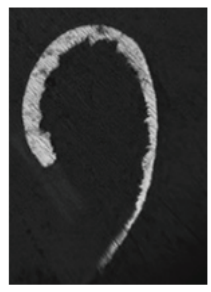

b

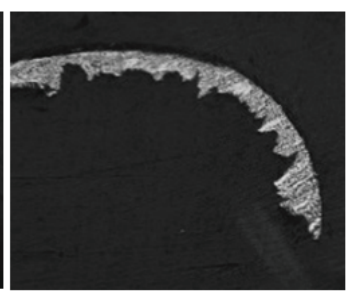

c
Fig. 1. Polished chips when processing VT-6 titanium alloy at the modes $\mathrm{V}=60 \mathrm{~m} / \mathrm{min}, \mathrm{t}=0.3 \mathrm{~mm}, \mathrm{~S}=0.05 \mathrm{~mm} /$ tooth (a); $\mathrm{S}=0.1 \mathrm{~mm} /$ tooth (b); $\mathrm{S}=0.2 \mathrm{~mm} /$ tooth (c).
In this work the study of strain-stress state in case of a lateral milling has employed the method of imitational finite-element modeling using DEFORM 2D software unit. The treated model is shown in Fig. 2.

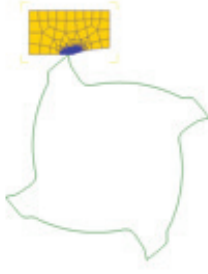

a

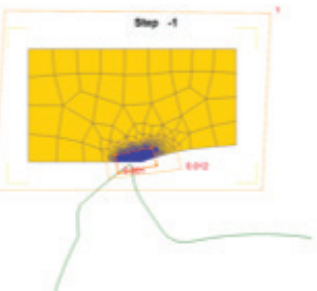

$\mathrm{b}$
Fig. 2. Modeling of the milling process using DEFORM 2D: a $2 \mathrm{D}$ model of the milling process; and $\mathrm{b}-$ areas of the grid condensation.

In the workpiece models they have made a cross section with the depth of $0.3 \mathrm{~mm}$ (Fig. 2a) which imitates the surface after operation of the previous tooth. This operation has been introduced with the purpose of reducing the time of calculation due to the optimal generation of the grid using the software suite. The tool is viewed by the model as an absolutely rigid body. They use the analog of VT-6 - Ti6Al4V titanium alloy. In order to limit loss of the volume it is necessary to condense the grid where a crack is expected to occur. The areas of the finite-element grid condensation: The internal area - cell size of $0.001 \mathrm{~mm}$, average $-0.012 \mathrm{~mm}$, external $-1 \mathrm{~mm}$ (Fig. 2b). The temperature of the tool and workpiece equals $20^{\circ} \mathrm{C}$. The constant friction ratio (according to Siebel) of the tool and the workpiece is 0.12 ; and the constant friction ratio of chips and the workpiece is 0.5 . Heat exchange with the environment is not taken into account.

\section{Segmentation process modeling}

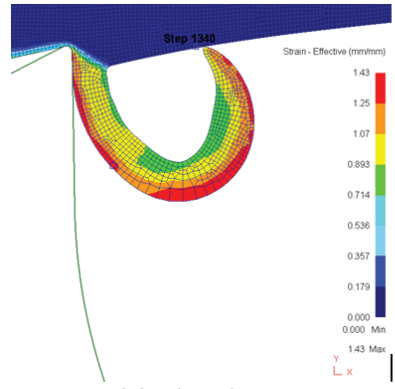

Critical Value $=0$

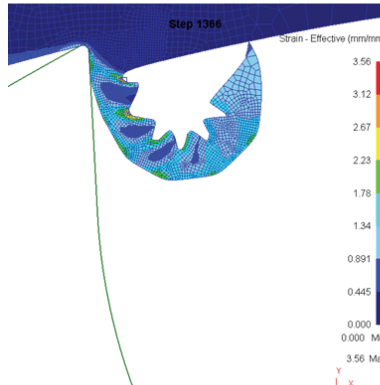

Critical Value $=300$

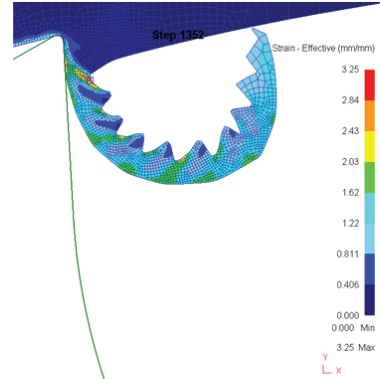

Critical Value $=150$

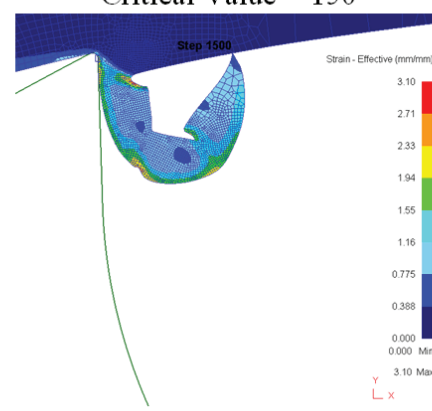

Critical Value $=500$

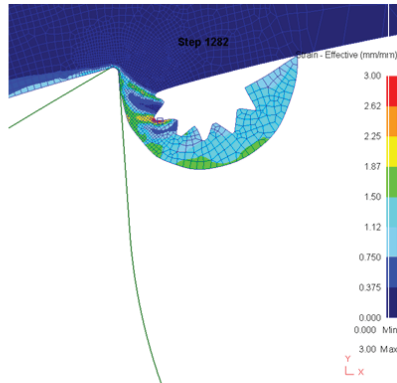

Critical Value $=230$

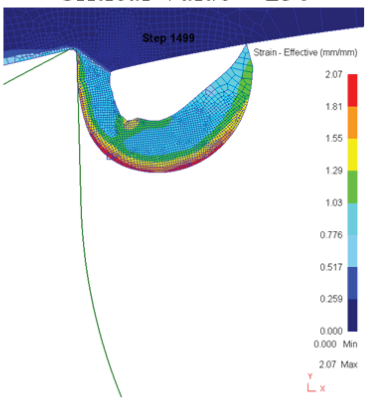

Critical Value $=800$

Fig. 3. Impact of the critical value of the destruction criterion on the chip morphology. 
One of the indicators of evaluation of the adequacy of imitational modeling is the chip morphology. Adequacy of imitational modeling using the chip morphology criterion depends on the choice of the corresponding destruction criterion which defines the moment of the material's exhaustion of its plasticity reserve in the process of deformation. Cockroft-Latham law has been chosen to describe destruction of the material. This criterion is empirical therefore the adequacy of this criterion depends on the limit value of plastic deformations at which the material begins to flow.

\section{Discussion of the study results}

In order to evaluate the adequacy of the models developed they have analyzed the impact of the critical value of destruction on the process of chip segmentation. In Fig. 3 they different types of chips are shown as achieved in the process of modeling for the following treatment modes: $\mathrm{V}=60 \mathrm{~m} / \mathrm{min}, \mathrm{S}=0.2 \mathrm{~mm} / \mathrm{rev}$., $\mathrm{t}=0.3 \mathrm{~mm}$ depending on the critical value of $\mathrm{CL}$ destruction. In the process of modeling the value of critical value of CL destruction has varied within the range from 0 to 800 .

As shown in Fig. 3 when the critical value equals 0 chips have homogeneous features and as the criterion value increases the chips gain segmented shape. The period of formation of the segment increases all the way until the critical value which equals 200, and then it begins to drop. At the value of 500 the segments acquire incorrect shape and the chip thickness notably increases. When the value is above 800 the segment degenerated into two elongated parts with a sharp differential of thickness between them. Noteworthy, the frequency and type of generation of segmentation has a direct impact on the cutting force $\mathrm{F}_{\text {pes}}$.
The graphs of changes of the components of the cutting force overtime depending on the values of the critical value of the Cockroft-Latham criterion are shown in Fig. 4. As shown in Fig. 4 the cutting forces get reduced as the critical value increases until it reaches the value of 230 , and then they observed a growth of forces and when the critical value reaches 800 the cutting forces reach their maximum values, and afterwards they practically do not fluctuate.

In order to evaluate the adequacy of the models developed they have analyzed and compared the results of the numerical and field tests of the chip morphology. Fig. 5 shows the images of a chip cross cut for the cutting modes: $\mathrm{V}=60 \mathrm{~m} / \mathrm{min}, \mathrm{t}=0.3 \mathrm{~mm}$, and the three different loads: $\mathrm{S}=0.05 ; 0.1 ; 0.2 \mathrm{~mm} / \mathrm{rev}$., with the specified typical geometric parameters for the experiment and finiteelement modeling. For finite-element models - based on the received geometrical parameters of the chips - they have selected the models which had been calculated using critical values of destruction criterion which equaled 300 . As shown in

Fig. 5 the obvious sawtooth configuration of the cross cut of the chips is only typical for the load $\mathrm{S}=0.2 \mathrm{~mm} / \mathrm{rev}$., both for the numerical study and the experimental one. For the load $\mathrm{S}=0.1 \mathrm{~mm} / \mathrm{rev}$. evaluation of the segmentation using geometrical method is complicated as the height of dents of the segments and their depth are depths vary, on average, by $6 \mathrm{mkm}$. For the load $\mathrm{S}=0.05$ $\mathrm{mm} / \mathrm{rev}$. the cross cut of the chips gains distorted shape from the standpoint of evaluation of the segmentation and in terms of its shape it is rather homogeneous and the only parameter which is used to typify it is the crosscut's average thickness. It should be noted that the result of simulation with a rather high degree of accuracy predicts the shape of chips for all three modes.
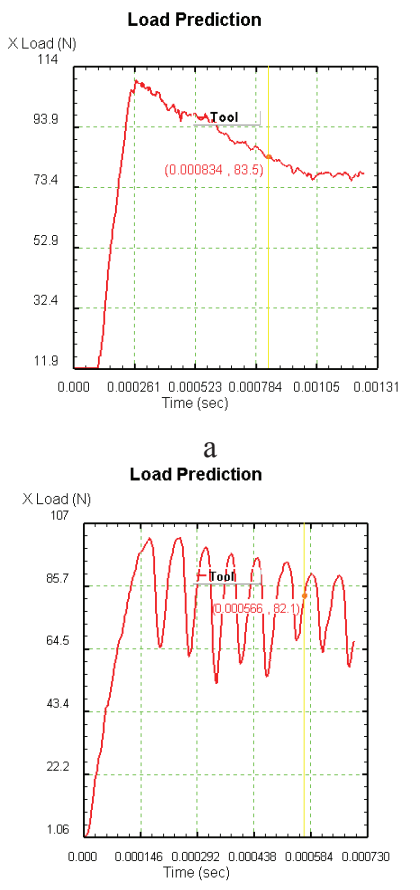

d

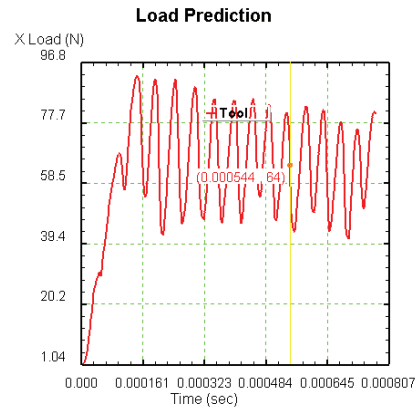

b

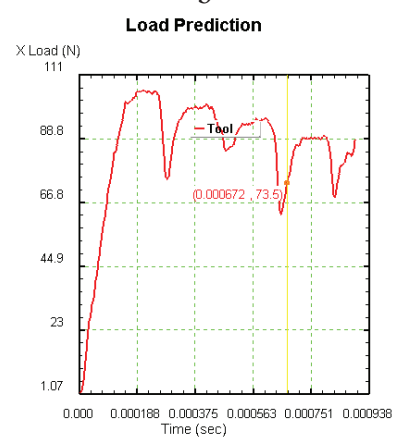

e

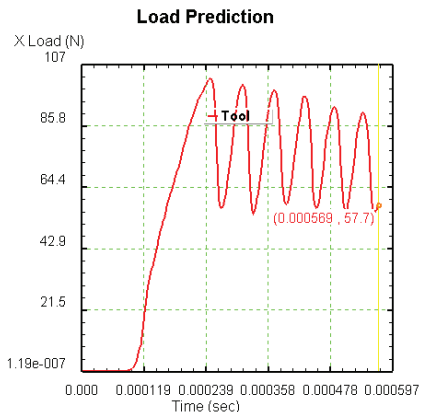

$\mathrm{C}$

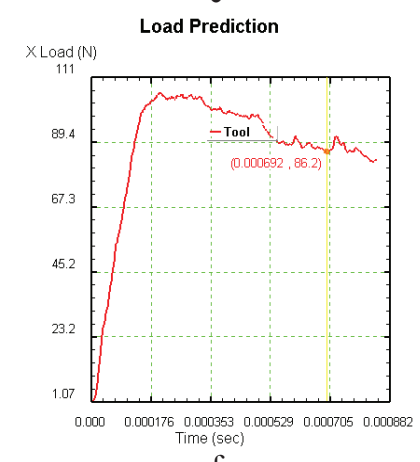

f

Fig. 4. Impact of the destruction on average cutting force at the critical values: a-0, b-150, c-230, d-300, e-500, e-800. 

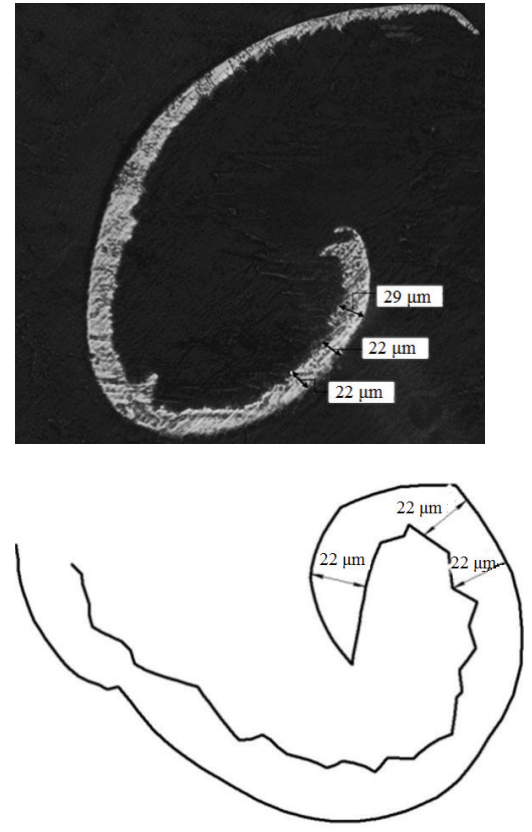
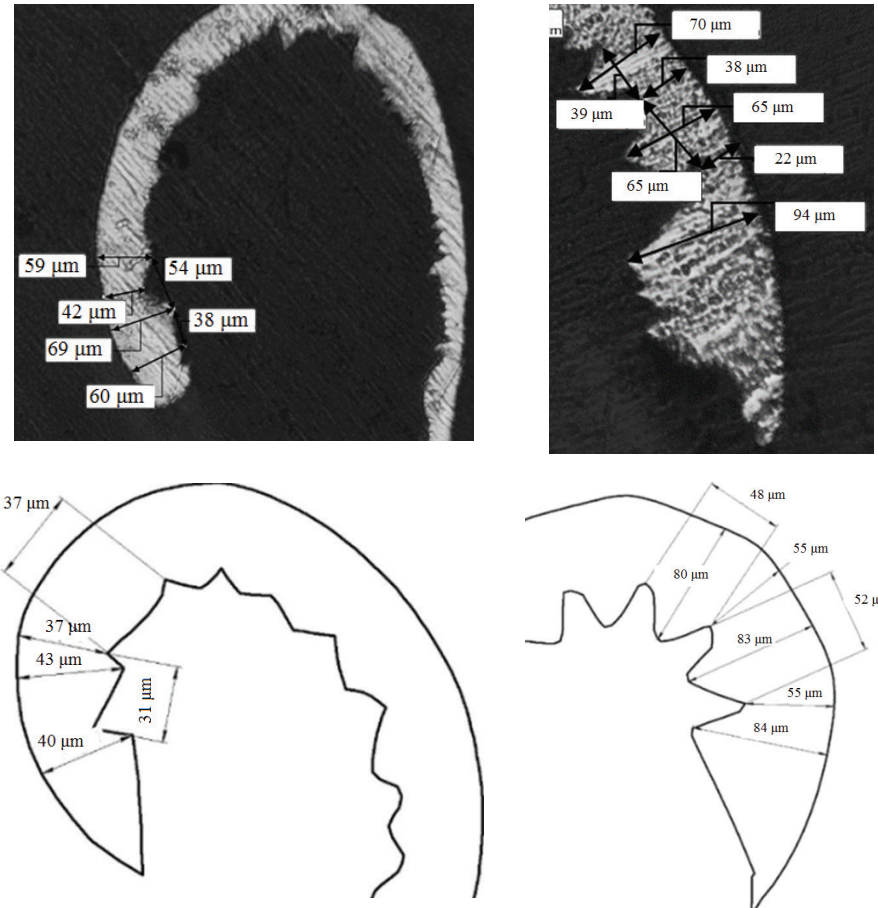

$\mathrm{b}$

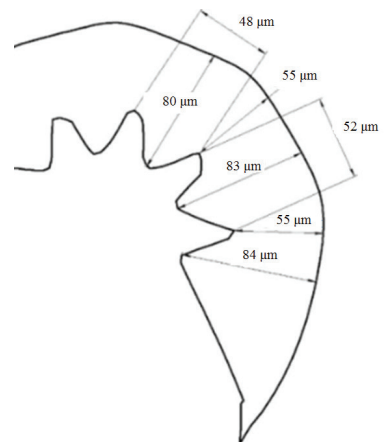

$\mathrm{c}$

Fig. 5. Cross section of a chip cut for cutting modes, $V=60 \mathrm{~m} / \mathrm{min}, \mathrm{t}=0.3 \mathrm{~mm} \mathrm{~S}=0.05 \mathrm{~mm} / \mathrm{rev}$. (a); $0.1 \mathrm{~mm} / \mathrm{rev}$. (b); $0.2 \mathrm{~mm} / \mathrm{rev}$. (c) for the experiment and finite-element modeling.

As shown in Fig. 5 the obvious sawtooth configuration of the cross cut of the chips is only typical for the load $\mathrm{S}=0.2 \mathrm{~mm} / \mathrm{rev}$, both for the numerical study and the experimental one. For the load $\mathrm{S}=0.1 \mathrm{~mm} / \mathrm{rev}$. evaluation of the segmentation using geometrical method is complicated as the height of dents of the segments and their depth are depths vary, on average, by $6 \mathrm{mkm}$. For the load $\mathrm{S}=0.05 \mathrm{~mm} / \mathrm{rev}$. the cross cut of the chips gains distorted shape from the standpoint of evaluation of the segmentation and in terms of its shape it is rather homogeneous and the only parameter which is used to typify it is the crosscut's average thickness. It should be noted that the result of simulation with a rather high degree of accuracy predicts the shape of chips for all three modes.

In Table 1 the cutting force values are shown as achieved in the process of experimental studies and the numerical modeling $(\mathrm{CL}=300)$ in the process of their treatment at the modes $\mathrm{V}=60-80 \mathrm{~m} / \mathrm{min}, \mathrm{t}=0.3 \mathrm{~mm}$, and the three various loads: $\mathrm{S}=0.05 ; 0.1 ; 0.2$.

Table 1. Cutting forces in the process of treatment of VT-6 titanium alloy

\begin{tabular}{|c|c|c|c|c|c|c|}
\hline \multirow{4}{*}{ 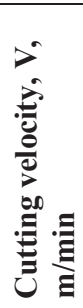 } & \multicolumn{6}{|c|}{ Load S, mm/tooth } \\
\hline & \multicolumn{2}{|r|}{0.05} & \multicolumn{2}{|c|}{0.1} & \multicolumn{2}{|r|}{0.2} \\
\hline & \multicolumn{6}{|c|}{ Cutting force $\mathrm{F}, \mathrm{H}$} \\
\hline & $\begin{array}{l}\stackrel{00}{\Xi} \\
\frac{0}{0} \\
\frac{0}{\Sigma}\end{array}$ & 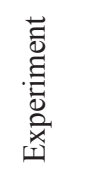 & $\begin{array}{l}\stackrel{\infty}{\Xi} \\
\frac{0}{0} \\
\stackrel{0}{\Sigma}\end{array}$ & 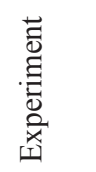 & 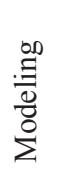 & 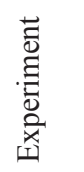 \\
\hline 60 & 35.64 & 34.68 & 52.25 & 54.72 & 84.4 & 86.42 \\
\hline 80 & 36.37 & 37.88 & 52.5 & 56.79 & 82.9 & 90.72 \\
\hline
\end{tabular}

As apparent from Table 1 the experimental data of the cutting force are well harmonized with the data of numerical modeling with the ratio error of no more than $5 \%$. For more adequate results it is required to further refine the data using rheological properties of the material during its treatment.

\section{Conclusion}

1) An imitation model for the cutting process has been developed using DEFORM 2D software suite;

2) Adequacy of the model developed based on the comparison of cutting forces and the shape of chips acquired in the process of the experiment and the modeling has been validated;

3) The $2 \mathrm{D}$ modeling data are well harmonized with the experimental data, while the error of forecasting the cutting force does not exceed the value of $5 \%$. More a more adequate result it is required to further refine the data using rheological properties of the material in the process of its treatment.

4) The imitational model developed for lateral milling which includes experimentally verified values of the Cockroft-Latham destruction criteria is applicable for solving the tasks of optimization of the geometry of cutting tools and operating parameters of cutting.

This work has enjoyed the support of the Ministry of Education and Sciences of the Russian Federation under the framework of the « Research and Development of Priority Areas of Scientific and Technical Complex of the Russian Federation in 2014$2020 »$ Program. 


\section{References}

1. N.I. Klushin, Metals Cutting Publishing House, (1958)

2. A.I. Khaimovich, Self-construction. Problems and prospects, 402 (2011)

3. M.A. Davies, Y. Chou, C.J. Evans, Natl. Inst. of Std. and Technology, (1996)

4. S. Atlati, B. Haddag, M. Nouari, M. Zenasni, International Journal of Machine Tools and Manufacture 51, 9 (2011)

5. A.N. Afonin, Modeling of destruction of metals by plastic deformation in the DEFORM and LC-DYNA, 25 (2007)

6. D.V. Evdokimov, D.L. Skuratov, D.G. Fedorov, Russian Engineering Research 36, 4 (2016).

7. M.G. Cockroft. D.J. Latham, J. Institute of Metals, 96 (1968)

8. A.N. Zhidyaev. M.A. Bolotov. I.L. Shitarev, Vestnik Rybinsk State Aviation Technological Academy P.A. Solovyov, (2010)

9. M.A. Bolotov, V.A. Pechenin, Journal of Materials and Environmental Science 7, 9 (2016)

10. R.H Komanduri, I. Brown, Journal of Engineering for Industry, 103 (1981)

11. D.L. Skuratov, A.N. Zhidyaev, M.B. Sazonov, Res. J. of Appl. Sci. 9, 11 (2016)

12. T. Thepsonthi. T. Ozel, Journal of Materials Processing Technology, 213 (2013)

13. T. Thepsonthi. T. Ozel, Journal of Materials Processing Technology, 221 (2015)

14. M. Galamaz. D. Coupard. F. Girot, International Journal of Machine Tools and Manufacture, 48 (2008)

15. V.P. Alekseev, A.I. Khaimovich, Key Engineering Materials, 684 (2015) 